\title{
Caregiver Attitudes and Hospitalization Risk in Michigan Residents Receiving Home- and Community-Based Care
}

\author{
Lisa R. Shugarman, PhD, * Amna Buttar, MS, MBBS, $+\neq \|$ Brant E. Fries, PhD, \$q \\ Tisha Moore, BA, and Caroline S. Blaum, MD, MS ${ }^{\sharp \#}$
}

OBJECTIVES: To study a cohort of participants in homeand community-based services (HCBS) in Michigan to evaluate the relationship between (1) caregiver attitudes and participant characteristics and (2) the risk of hospitalization. DESIGN: A population-based study.

SETTING: HCBS programs funded by Medicaid or state/ local funds in Michigan.

PARTICIPANTS: Five hundred twenty-seven individuals eligible for HCBS in Michigan were studied. These HCBS participants were randomly selected clients of all agencies providing publicly funded HCBS in Michigan from November 1996 to October 1997.

MEASUREMENTS: Data for this study were collected using the Minimum Data Set for Home Care. Assessments were collected longitudinally, and the baseline (initial admission assessment) and 90-day follow-up assessments were used. Key measures were caregiver attitudes (distress, dissatisfaction, and decreased caregiving ability) and HCBS participant characteristics (cognition, functioning, diseases, symptoms, nutritional status, medications, and disease stability). Multinomial logistic regression was used to evaluate how these characteristics were associated with the competing risks of hospitalization and death within 90 days of admission to HCBS.

RESULTS: We found a strong association between caregiver dissatisfaction (caregiver dissatisfied with the level of care the home care participant was currently receiving) and an increased likelihood of hospitalization. HCBS participant

From the "RAND Corporation, Santa Monica, California; ${ }^{\dagger}$ Department of Internal Medicine, Indiana University School of Medicine, Indianapolis, Indiana; 沼diana University Center for Aging Research, Indianapolis, Indiana; SInstitute of Gerontology and "School of Public Health, University of Michigan, Ann Arbor, Michigan; "Ann Arbor VA Medical Center, Ann Arbor, Michigan; and "Department of Internal Medicine, University of Michigan Medical School, Ann Arbor, Michigan.

Support for this paper was provided by a grant from the Robert Wood Johnson Foundation and by interRAI, an international group of clinicians and researchers who collaborate to promote research on the assessment instruments and quality outcomes for older people.

An early version of this paper was presented at the 1999 Annual Meeting of the Gerontological Society of America in San Francisco, California.

Address correspondence to Lisa R. Shugarman, PhD, RAND Corporation, 1700 Main Street, P.O. Box 2138, Santa Monica, CA 90407.

E-mail: lisa_shugarman@rand.org. cancer, chronic obstructive pulmonary disease, pain, and flare-up of a chronic condition were also associated with increased hospitalization. Poor food intake and prior hospitalization were associated with hospitalization and death.

CONCLUSIONS: We conclude that, within a cohort of people receiving HCBS who are chronically ill, highly disabled, and at high risk for hospitalization and death, interventions addressing caregiver dissatisfaction, pain control, and medical monitoring should be evaluated for their potential to decrease hospitalization. J Am Geriatr Soc 50: 1079-1085, 2002.

Key words: home- and community-based services; risk factors for hospitalization; caregiver characteristics

$\mathrm{T}$ he past 2 decades have seen a tremendous growth in home- and community-based services (HCBS) for older and disabled people. According to the Medical Expenditure Panel Survey, approximately 2.5 million people received home care services in $1996 .{ }^{1}$ The majority of these patients were aged 65 and older, ${ }^{2}$ and, of persons 85 and older, a full $31 \%$ were receiving home care services. ${ }^{3}$ Older and disabled individuals who enter HCBS often have care needs that are both acute and chronic, medical and personal. For many of the services provided, therefore, the major payors are Medicaid, designed for chronic and personal care services, and Medicare, designed for medical and acute, subacute, or rehabilitative care needs. ${ }^{3}$

Because users of HCBS have chronic diseases and disabilities, their use of medical services, such as physician visits and hospitalizations, is high. The relationship between HCBS and healthcare utilization, especially hospitalization, is still not well understood, despite the attention given to this area of study. Although it was hoped that HCBS could decrease the need for hospitalization, the precise group of people that would be expected to have high utilization uses HCBS services. Many studies have confirmed their high rate of hospitalization. ${ }^{4,5}$

Several studies, including the only randomized trial of HCBS, have shown that HCBS improved the quality of life and satisfaction of caregivers. ${ }^{6,7}$ Few studies have exam- 
ined the association between caregiver characteristics and the increased risk of hospitalization in disabled people in the community ${ }^{8}$ and none has looked specifically at people receiving HCBS. The goal of this research was to identify HCBS participant characteristics associated with an increased risk of hospitalization within a cohort of people receiving HCBS through publicly funded programs run by the state of Michigan. We specifically evaluated the hypothesis that caregiver characteristics, such as level of stress and perceived burden, would be associated with increased hospital use by HCBS participants, controlling for other known or hypothesized risks. We tested this hypothesis using the framework of the Andersen Behavioral Model for health services utilization, ${ }^{9}$ which allowed us to control for variables that previous research has demonstrated is associated with hospital use by HCBS clients.

These analyses were based on a unique data source of a cohort of individuals participating in publicly funded HCBS programs in Michigan. HCBS participants were assessed using the Minimum Data Set for Home Care (MDS$\mathrm{HC}$ ), an instrument designed to assess multiple domains of health and service/therapy use by HCBS participants, including function, symptoms, medical diagnoses, cognition, affective state, caregiver burden, and stress (sample and further instrument description below).

\section{METHODS}

\section{HCBS in Michigan}

Data for this study came from two programs operated by the state of Michigan that were designed to expand the availability of HCBS to older and disabled people: the Care Management (CM) Program and the Medicaid Home and Community-Based Services Waiver (the Waiver). Michigan has been operating the CM program since 1983. HCFA approved the Waiver in 1992 for a 3-year period, and extended and expanded it statewide in 1998. The population that the Waiver and CM programs serve is primarily older $(\geq 60)$, although the Waiver program also serves younger disabled populations. While both programs use the same functional eligibility criteria, the Waiver program also requires that participants meet the expanded financial eligibility criteria for Medicaid-funded nursing home care.

\section{Minimum Data Set for Home Care}

Data were collected using the MDS-HC, a multidimensional assessment instrument. (See Morris, et al. ${ }^{10}$ for a complete description of the development and reliability testing of the MDS-HC.) The MDS-HC was developed to be the community-based analogue to the nationally mandated MDS for nursing homes. ${ }^{10}$ The MDS-HC is a broad-based instrument incorporating multiple clinical and functional domains important for care planning, such as activities of daily living (ADLs), instrumental activities of daily living (IADLs), measures of cognition, disease diagnoses, and service utilization. This assessment is designed to use all possible sources of information (e.g., HCBS participants, caregivers, direct observation, medical records) to determine the most appropriate response for each assessment item. The data used for this study were collected to develop a screening algorithm to determine eligibility for HCBS, and the state has now adopted the MDS-HC as the basis for assessment and care planning in its HCBS programs.

\section{Study Subjects}

Study subjects were HCBS participants from the 14 regional agencies operating the Waiver or CM programs between November 1996 and October 1997. They were recruited into the study during each agency's normal screening process. In the smaller agencies, every potential participant screened for eligibility was asked to be involved in the study; in the larger agencies, every other potential participant was asked. Six hundred twenty-eight individuals found eligible for home care services through either the Waiver or CM programs agreed to participate in the study (more than $80 \%$ of those asked).

Multiple MDS-HC assessments were completed for each study participant. The baseline assessment was completed within 4 calendar days of the agency's initial assessment, to ensure that the client's condition did not change. Follow-up assessments were completed at 45, 90, and 180 days after the baseline assessment for most study participants. To preserve the sample size, we limited our analyses in this study to the baseline assessment for client characteristics and the 90-day follow-up assessments to determine whether the HCBS participant had been hospitalized after admission to home care.

Of the 628 individuals recruited into the study, 101 $(16.1 \%)$ were excluded in the analytic data set because they failed to complete the study protocol for reasons other than death. Reasons for exclusion were: loss to follow-up ( $\mathrm{n}=25)$, refusal to continue participating in the study $(\mathrm{n}=19)$, missing assessments $(\mathrm{n}=21)$, and discharge before the 90-day assessment $(\mathrm{n}=36)$. The final analytic data set for analyses of the competing risks of hospitalization and death contained 527 cases.

\section{Conceptual Framework}

Almost 30 years ago, Andersen et al. ${ }^{9}$ developed a behavioral model to explain why people use health services. The model suggested that health services utilization could be understood as a function of three categories of population characteristics: predisposing characteristics (demographic and health beliefs), enabling characteristics (individual and community resources), and need characteristics (perceived or evaluated health status, disability, and disease diagnoses). Since the development of this model, it has been widely applied to guide and instruct research on the factors related to healthcare utilization for older people and other populations. ${ }^{11-15}$

Bass et al. ${ }^{12}$ contend that the Andersen model fails to address the role of informal caregivers in older individuals' use of health services. They argue that caregivers influence older people's use of services directly by acting as a gatekeeper or case managers for accessing services and indirectly by influencing the older people's perceptions of need. Therefore, this study included three caregiver characteristics that may influence or enable the likelihood of hospitalization of the HCBS participant. Variables were chosen based on this model as follows (all characteristics are participant characteristics except where explicitly noted to be caregiver characteristics): 
1. Predisposing characteristics: gender, age, educational attainment.

2. Enabling characteristics: marital status, caregiver characteristics: (a) caregiver health problems that limit caregiver involvement; (b) caregiver dissatisfaction; (c) caregiver's expression of distress, anger, or depressive symptoms.

3. Need characteristics: heart/circulatory diagnoses, neurological diagnoses, musculoskeletal diagnoses, pneumonia, cancer, diabetes mellitus, chronic obstructive pulmonary disease (COPD), urinary tract infection (UTI), renal failure, pressure ulcer, pain, depression/anxiety symptoms, poor self-reported health, report of a recent flare-up of a chronic condition, decreased cognitive performance, ADL/IADL functioning, prior hospitalizations.

\section{Measurement of Variables}

The primary outcome of interest in these analyses was hospitalization after admission to HCBS. In addition, we included as an outcome whether the HCBS participant died before the 90-day follow-up assessment. For some of the individuals who died, a hospitalization may have preceded death, but that information was not available. To partially correct for this, we analyzed death as a competing risk of hospitalization. Our research goal was not to specify a mortality model in this HCBS sample but to determine whether those who died were similar to or different from those who were hospitalized but survived to the 90-day assessment.

Independent variables were measured using items from the MDS-HC. Most variables were taken from a single MDS item, but the measures of diagnoses, cognition, depressive symptoms, and ADL functioning were composites of several MDS questions. The heart/circulatory diagnoses included congestive heart failure, coronary artery disease, irregularly irregular pulse, and peripheral vascular disease. The musculoskeletal diagnoses included arthritis, hip or other fracture, and osteoporosis. The neurological diagnoses included Alzheimer's disease, dementia other than Alzheimer's, head trauma, multiple sclerosis, and Parkinsonism. The pain variable was defined by the presence of daily pain in the 7 days before assessment. Participants were defined as having a nutrition problem if, in the 3 days preceding assessment, a client consumed little or no food or fluid, or less than usual. Polypharmacy was defined as taking six or more medications on a regular basis in the 7 days before assessment.

Cognitive functioning was classified according to the Cognitive Performance Scale (CPS), which summarizes the measures of cognition from the MDS-HC in a single, functionally meaningful, seven-category (0-6) hierarchical measure. ${ }^{16}$ The CPS values represent cognitive functioning ranging from intact (0) to very severely impaired (6). The CPS has been validated against and shown to be predictive of the Folstein Mini-Mental State Examination. ${ }^{16,17}$ Those with CPS values of 5 or 6 were identified as having severely impaired cognitive performance.

The depression/anxiety symptoms variable was based on six MDS-HC items. It included behaviors observed by the care manager or others that might indicate depressed affect, such as crying, persistent anger with self or others, repetitive anxious complaints, sad or pained facial expressions, withdrawal from activities of interest, and thinking about death. ${ }^{18}$ Clients were considered to exhibit depressive or anxiety-related symptoms if they presented with one or more of these indicators at baseline.

Physical functioning was measured as a sum of eight dichotomized ADLs (bed mobility, transferring, locomotion, dressing, eating, toilet use, personal hygiene, and bathing) and seven dichotomized IADLs (meal preparation, ordinary house work, managing finances, managing medications, phone use, shopping, and transportation), giving a total physical functioning scale ranging in value from 0 to 15 . ADLs were dichotomized to reflect those independent or requiring supervision only versus all others. IADLs were dichotomized to reflect those who were independent in the task versus all others. We considered ADL and IADL scales separately in our models (data not shown) but ultimately decided to proceed with a single scale. To be eligible for HCBS in Michigan, one must be eligible for nursing home level of care. As such, the study population was already impaired at admission to HCBS, and we did not expect much variation in the population due to functioning. The ADL and IADL scales were highly collinear, and considering them separately did not significantly improve our models. The resulting combined scale was consistent with the work of Spector et al. ${ }^{19}$ in which the authors found that a single scale measure of ADLs and IADLs was appropriate for measuring physical functional disability. We included the ADL/IADL measure as a continuous variable in our analyses.

Included among the enabling characteristics were three measures of caregiver attitude regarding perceived burden hypothesized to be associated with an increased risk of hospitalization among home care clients. The literature on caregiver burden distinguishes between two different types of burden: personal and interpersonal. ${ }^{20}$ Personal burden reflects the caregiver's appraisal of limitations on the caregiver's activities as a result of providing care to the impaired person, whereas interpersonal burden is a reflection of the problems the caregiver has with the impaired individual's condition.

The measures of caregiver attitude in the MDS-HC were chosen to reflect both types of burden. These measures were incorporated into the assessment to evaluate whether the support system was capable of meeting the needs of the HCBS participant. ${ }^{21}$ The caregiver measures were defined as follows: (1) the caregiver was unable to continue in caring activities due to declines in his/her own health; (2) the caregiver expressed feelings of distress, anger, or depression; and (3) the caregiver was dissatisfied with the support received from family and friends. Measures 1 and 2 may be considered measures of personal burden, whereas Measure 3 may be considered interpersonal burden.

Additional (participant) variables included in our analyses were gender, age (continuous), educational attainment (high school education or less vs higher education), marital status (married vs not married), poor selfreported health, report of a recent flare-up of a chronic condition, and prior hospitalizations. All variables were dichotomous variables unless otherwise noted. 


\section{Analytic Plan}

Bivariate analyses were conducted to identify significant associations between the independent variables and the dependent variable of interest: hospitalization and its competing risk, death. Cross-tabulations were constructed and chi-square statistics calculated for dichotomous and categorical independent variables, and analysis of variance was employed for continuous independent variables. Variables found to be significantly associated with the outcome variables at $P<.10$ in the bivariate case were included in further multivariate analyses.

Multivariate analyses were conducted with a multinomial logistic model to model the competing risks of hospitalization and death. Rather than estimate a series of binomial logistic models, these outcomes were estimated concurrently, which maximized the efficiency of the $\beta$ coefficients. Results are presented as local odds ratios and $90 \%$ confidence intervals, describing the odds of the particular outcome relative to the reference category (remaining in home care without hospitalization or death for 90 days).

\section{RESULTS}

Table 1 describes the characteristics of the HCBS sample. Almost $16 \%(n=84)$ of HCBS participants had one or more hospitalizations, and $7.0 \%(\mathrm{n}=37)$ died within 90 days of admission to HCBS. The majority of the HCBS sample was female $(69.1 \%)$, with an average age of 75 ; $92.6 \%$ were aged 60 and older. Slightly more than onethird of the sample population was married $(35.5 \%)$, and $18.4 \%$ of the study participants had 12 or fewer years of education. Those who were severely cognitively impaired (CPS of 5 or 6) constituted $10.1 \%$ of the total sample, and the mean number of total ADL and IADL impairments combined was 6.0 (range $0-15$ ).

Table 2 describes the significant bivariate associations of participant characteristics with hospitalization and death compared with the reference group (remaining in

\begin{tabular}{lc}
\hline \multicolumn{1}{c}{ Table 1. Sample Characteristics $(\mathbf{N}=527)$} \\
\hline \multicolumn{1}{c}{ Characteristic } & $\begin{array}{c}\text { Total } \\
\text { Sample }\end{array}$ \\
\hline Female, \% & 69.1 \\
Married, \% & 35.5 \\
High school or less, \% & 18.0 \\
Severe cognitive impairment, \%* & 10.1 \\
Prior hospitalizations, \% & 45.7 \\
Age, mean \pm SD & $75.2 \pm 11.6$ \\
ADL/IADL impairments, mean \pm SD $^{\dagger}$ & $6.0 \pm 3.6$ \\
Outcome & \\
Remained in Home Care, \% & 77.0 \\
With $\geq 1$ Hospitalizations in HCBS, \%\$ & 15.9 \\
Died, \% & 7.0 \\
\hline
\end{tabular}

\footnotetext{
${ }^{\star}$ Percentage of those with a score of 5 or 6 on the Cognitive Performance Scale.

+Sum of all impairments, range $0-15$.

₹Total less than $100 \%$ because of rounding.

In the first 90 days after admission.

$\mathrm{SD}=$ standard deviation; $\mathrm{HCBS}=$ home and community-based services; ADL $=$ activities of daily living; IADL $=$ instrumental activities of daily living.
}

HCBS with no hospitalizations). The associations determined to be statistically significant with one or both outcomes at $P<.10$ are presented in this table. Older age was significantly associated with death but not hospitalization. Women were significantly less likely to be hospitalized than to remain in HCBS. Being married was significantly associated with hospitalization. Two of the caregiver attitude measures were significantly associated with the outcomes, but their associations were not consistent. Caregiver dissatisfaction with the current level of support received by the client was significantly associated with hospitalization, whereas caregiver distress was significantly associated with death.

Disease diagnoses and other clinical characteristics also had mixed associations. Heart conditions were significantly associated with an increased likelihood of hospitalization, whereas neurological conditions were protective against hospitalization. Musculoskeletal conditions were associated with a decreased likelihood of death. Cancer and renal diseases were significantly associated with hospitalization and death. Prior hospitalization and poor food intake were significantly associated with both outcomes, whereas pain, polypharmacy, and experiencing a flare-up of a chronic condition were associated with hospitalization only. Variables not found to be statistically significant with either outcome were educational attainment, pneumonia, diabetes mellitus, UTI, depression/anxiety symptoms, and poor self-reported health.

Table 3 gives results from the multinomial logistic regression model predicting hospitalization or death within 90 days. This table shows odds ratios relative to the reference group for each predictor ordered by their relative magnitude of association with the likelihood of hospitalization. The model controlled for predisposing characteristics (age, gender, educational level), cognitive performance, and ADL/IADL impairments (not shown).

In general, different enabling and need characteristics were associated with each of the two outcomes. The only characteristics positively and significantly associated with both hospitalization and death were poor food intake and prior hospitalization. The characteristic most strongly associated with an increased likelihood of hospitalization was caregiver dissatisfaction, even after controlling for key chronic diseases and other potentially important clinical variables. Also associated with increased odds of hospitalization were being married, experiencing a flare-up of a chronic condition, experiencing pain on a daily basis in the week before assessment, and having a diagnosis of cancer or COPD. Having a neurological problem (Alzheimer's disease or other dementia) was protective against hospitalization in the study sample. Characteristics associated with death only were presence of renal disease, whereas the presence of a musculoskeletal disease was protective.

\section{DISCUSSION}

This study is one of the few to evaluate the effect of caregiver characteristics on hospitalization in a sample of disabled individuals receiving home- and community-based care that controlled for key clinical characteristics. Although caregiver characteristics have been shown to be associated with hospitalization use in previous studies, the strength of the association in our study is surprising, especially because other relevant and significant clinical char- 
Table 2. Bivariate Associations of Participant Characteristics with Hospitalization and Death Compared with HCBS

\begin{tabular}{|c|c|c|c|}
\hline \multicolumn{4}{|l|}{ Predisposing characteristics } \\
\hline Female, \% & 71.4 & $60.7^{\star \star}$ & 62.2 \\
\hline Age, mean $\pm S D$ & $75.2 \pm 11.9$ & $74.6 \pm 11.0$ & $76.8 \pm 9.1^{\star}$ \\
\hline \multicolumn{4}{|l|}{ Enabling characteristics, \% } \\
\hline Caregiver distressed/angry/depressed & 20.3 & 19.5 & $35.1^{\star *}$ \\
\hline \multicolumn{4}{|l|}{ Need characteristics } \\
\hline $\mathrm{ADL} / \mathrm{IADL}$ impairments, mean $\pm \mathrm{SD}^{\dagger}$ & $5.81 \pm 3.5$ & $6.2 \pm 3.2$ & $7.9 \pm 4.0^{\star \star \star}$ \\
\hline Cognitive performance scale, $\%^{\ddagger}$ & 8.4 & 13.1 & $21.6^{\star \star \star}$ \\
\hline$\geq 6$ medications, $\%$ & 60.3 & $70.2^{*}$ & 67.6 \\
\hline Cancer, \% & 8.9 & $17.9^{\star \star}$ & $24.3^{\star \star \star}$ \\
\hline Poor food intake, \% & 17.7 & $33.3^{\star \star \star \star}$ & $41.7^{\star \star \star \star}$ \\
\hline Pressure ulcers, \% & 4.9 & $9.5^{\star}$ & $13.5^{\star \star}$ \\
\hline Renal failure, \% & 5.2 & $11.9^{\star \star}$ & $18.9^{\star \star \star \star}$ \\
\hline Flare-up of chronic condition, \% & 20.4 & $38.1^{\star \star \star \star}$ & 29.7 \\
\hline Prior hospitalizations, $\% \$$ & 40.1 & $65.5^{\star \star \star \star}$ & $62.2^{\star \star \star}$ \\
\hline
\end{tabular}

Note: Statistical significance noted for relationship between each outcome and the reference group (remaining in home and community-based services (HCBS)).

${ }^{\star} P<.10 ;{ }^{\star \star} P<.05 ;{ }^{\star \star \star} P<.01 ;{ }^{\star \star \star \star} P<.001$.

Thange: $0-15$.

₹Percentage of those with a cognitive performance scale score of 5 or 6 .

SAny hospitalization in the 90 days before the baseline assessment.

$\mathrm{SD}=$ standard deviation; $\mathrm{ADL}=$ activities of daily living; IADL = instrumental activities of daily living.

acteristics were also independently associated with increased hospitalization. The importance of caregiver dissatisfaction is particularly convincing because our study collected caregiver characteristics concurrently with clinical information, our methodology allowed for case-mix adjustment, and the study followed HCBS participants prospectively, accounting for relevant competing outcomes.

The particular caregiver characteristic that was most associated with hospital risk was the characteristic "caregiver not satisfied with support provided by others." Although this measure implies that the caregiver is unhappy with the lack of assistance from other caregivers, and has been considered a measure of interpersonal burden, it can also be viewed as a reflection of the caregiver's inability to provide for all the needs of the disabled person. In other words, the impaired individual has more needs than the primary caregiver can address alone.

This finding, along with our finding that being married is associated with increased hospitalization, is consistent with an "advocate" theory of health services utilization in disabled older people. A committed and highly involved caregiver may also be highly skilled in accessing formal care and medical care for the disabled older person. To test whether a dissatisfied caregiver might be dissatisfied because of particular clinical problems (e.g., pain, polypharmacy, or unstable condition), we tested for first-order interactions, but we found no significant relationships.

In bivariate analyses, caregiver distress (feeling excessive burden associated with caregiving) was significantly associated with risk of death, but this relationship was not observed in the multivariate analyses. The caregiver characteristic "unable to continue in caring activities due to declines in caregiver health was not associated with either outcome. This finding may be partly due to our study group, which was chronically ill, severely disabled, and in need of significant care. Any impaired or ill caregiver may have long since relinquished a caregiver role.

HCBS participant clinical characteristics were also associated with hospitalization and death. Prior hospitalization is well known to be associated with negative outcomes. ${ }^{8}$ Previous studies have also demonstrated that undernutrition is associated with hospitalization, morbidity, and death in nursing home residents, geriatric rehabilitation patients, and hospitalized older people,,$^{22,23}$ but this may be the first report of the association between poor oral intake and hospitalization and mortality in HCBS participants. The strong association between poor intake and death may be a reflection of some participants who were receiving comfort care only, but our data did not allow us to test for this possibility.

It is interesting to speculate about interventions designed to decrease hospitalization rates for people in home care, because several of the characteristics significantly associated with increased hospitalization in our study are potentially amenable to interventions. Pain can be closely monitored and treated. In addition, monitoring can be intensified to prevent hospitalization due to exacerbations of chronic conditions, although we need better information to be better able to anticipate changes and identify important markers of change in chronic conditions in the home care popula- 
Table 3. Characteristics Predicting Hospitalization or Death Within 90 Days in a Cohort of Home and Community-Based Services Clients-Multinomial Logistic Regression

\begin{tabular}{|c|c|c|c|c|}
\hline \multirow[b]{2}{*}{ Characteristic } & \multicolumn{2}{|c|}{ Hospitalized } & \multicolumn{2}{|c|}{ Died } \\
\hline & Odds Ratio & $90 \% \mathrm{Cl}$ & Odds Ratio & $90 \% \mathrm{Cl}$ \\
\hline Caregiver dissatisfied with care received & 2.99 & $1.38-6.46^{\star}$ & 1.77 & $0.60-5.28$ \\
\hline Cancer & 2.50 & $1.34-4.68^{*}$ & 2.02 & $0.83-4.87$ \\
\hline Prior hospitalizations & 2.41 & $1.48-3.90^{\star}$ & 2.47 & $1.23-4.97^{*}$ \\
\hline Married & 2.20 & $1.31-3.68^{*}$ & 1.70 & $0.80-3.61$ \\
\hline Flare-up of chronic condition & 1.98 & $1.17-3.33^{*}$ & 1.17 & $0.51-2.69$ \\
\hline Poor food intake & 1.89 & $1.10-3.25^{\star}$ & 2.60 & $1.24-5.44^{*}$ \\
\hline Daily pain & 1.76 & $1.10-2.84^{*}$ & 1.36 & $0.68-2.72$ \\
\hline Chronic obstructive pulmonary disease & 1.57 & $0.94-2.63$ & 0.47 & $0.18-1.28$ \\
\hline Heart/circulatory problems & 1.35 & $0.80-2.27$ & 0.65 & $0.31-1.34$ \\
\hline Musculoskeletal problems & 1.23 & $0.70-2.16$ & 0.42 & $0.20-0.87^{*}$ \\
\hline$\geq 6$ medications & 1.14 & $0.67-1.93$ & 1.42 & $0.68-2.97$ \\
\hline Renal failure & 0.92 & $0.37-2.30$ & 3.43 & $1.31-8.99^{*}$ \\
\hline Caregiver distressed/angry/depressed & 0.58 & $0.31-1.10$ & 0.86 & $0.38-1.95$ \\
\hline Neurological problems & 0.37 & $0.19-0.71^{*}$ & 0.66 & $0.30-1.45$ \\
\hline
\end{tabular}

Note: Reference group remained in home and community-based services without hospitalization.

Model controlled for age, gender, education level, impairments in activities of daily living and instrumental activities of daily living, and cognition.

${ }^{\star} P<.05$.

$\mathrm{CI}=$ confidence interval.

tion. Caregiver concerns can be identified, and support and counseling may be offered. Alternatively, caregiver dissatisfaction may be an indicator that the patient's needs exceed the ability of the caregiver/home care system to provide for them and that a higher level of care is warranted.

One of the challenges in designing interventions for this population will be bridging the divide created by the different funding sources that pay for chronic and acute care. Medicaid primarily pays for the HCBS programs described in this study, whereas hospital care is the domain of Medicare. As long as these two funders operate separately from one another, there will be little incentive for one program to invest dollars in interventions that are likely to save money in the other program.

The primary limitation of our study is that the data we used for this study were collected as part of a project to develop and evaluate a new system for screening potential home care clients into the state-funded programs. As a result, we knew whether a hospitalization had occurred within 90 days of the baseline assessment but had no additional information about such information as the timing of the hospitalization or length of stay. If someone went to a hospital and died, we were only aware of their death, not the intercurrent hospitalization. Nevertheless, the MDS-HC is now being used on an ongoing basis as a part of the state's standard assessment instrument, so more recent assessments may be coupled with hospitalization data. Future studies may also incorporate more-detailed information about the timing of health events for the HCBS population.

We studied HCBS participants served by an on-going statewide program where decisions are being made, disabled individuals are receiving care, and taxpayer money is being spent. This study shows how policy involving waiver programs and assessment instrument choices translates into care of a vulnerable population. It illustrates how the interrelationship of disease diagnoses, the natural history of disease, and caregiver attitudes affects utilization. We believe that our findings, even though they represent an HCBS program in one state, are intriguing and suggest the need for follow-up outcomes studies and practice-change interventions in home- and community-based care to increase caregiver support, offer aggressive pain management, and monitor for unstable disease.

\section{ACKNOWLEDGMENTS}

The authors wish to thank Mary James and the anonymous reviewers for their valuable comments and assistance on this paper.

\section{REFERENCES}

1. Krauss NA, Machlin S, Kass BL. Use of Health Care Services, 1996 (AHCPR publication no. 99-0018). Rockville, MD: Agency for Health Care Policy and Research, 1999.

2. Haupt BJ. An Overview of Home Health and Hospice Care Patients: 1996 National Home and Hospice Care Survey. Advance Data from Vital and Health Statistics, No 297. Hyattsville, MD: National Center for Health Statistics, 1998.

3. Health Care Financing Administration. National Health Expenditures Projections, 1998-2008. Health Care Financing Administration. Available: http: //www.hcfa.gov/stats/NHE-Proj/, Accessed: September 20, 2000.

4. Wooldridge J, Schore J. The evaluation of the National Long Term Care Demonstration 7 . The effect of channeling on the use of nursing homes, hospitals, and other medical services. Health Serv Res 1988;23:119-127.

5. Anderson MA, Helms LB, Hanson KS et al. Unplanned hospital readmissions: A home care perspective. Nurs Res 1999;48:299-307.

6. Kemper P. The evaluation of the National Long Term Care Demonstration 10. Overview of the findings. Health Serv Res 1988;23:161-174.

7. Hughes SL, Cummings J, Weaver F et al. A randomized trial of the cost effectiveness of VA hospital-based home care for the terminally ill. Health Serv Res 1992;26:801-817.

8. Miller EA, Weissert WG. Predicting elderly people's risk for nursing home placement, hospitalization, functional impairment, and mortality: A synthesis. Med Care Res Rev 2000;57:259-297.

9. Andersen R, Newman JF. Societal and individual determinants of medical care utilization in the United States. Milbank Q 1973;51:95-124. 
10. Morris J, Fries B, Steel K et al. Comprehensive clinical assessment in community setting: Applicability of the MDS-HC. J Am Geriatr Soc 1997;45:10171024.

11. Miller B, McFall S. The effect of caregiver's burden on change in frail older persons' use of formal helpers. J Health Soc Behav 1991;32:165-179.

12. Bass DM, Noelker LS. The influence of family caregivers on elder's use of inhome services: An expanded conceptual framework. J Health Soc Behav 1987;28:184-196.

13. Penning MJ. Health, social support and the utilization of health services among older adults. J Gerontol B Psychol Sci Soc Sci 1995;50B:330-339.

14. Fortinsky RH, Madigan EA. Home care resource consumption and patient outcomes: What are the relationships? Home Health Serv Q 1997;16:55-73.

15. Logan JR, Spitze G. Informal support and the use of formal services by older Americans. J Gerontol B Psychol Sci Soc Sci 1994;49B:S25-S34.

16. Morris JN, Fries BE, Mehr DR et al. MDS Cognitive Performance Scale. J Gerontol 1994;494:M174-M182.

17. Hartmaier SL, Sloane PD, Guess HA et al. Validation of the Minimum Data
Set Cognitive Performance Scale: Agreement with the Mini-Mental State Examination. J Gerontol A Biol Sci Med Sci 1995;50A:M128-M133.

18. Phillips CD, Morris JN, Mor V. Validating a mood scale based on the Minimum Data Set (MDS) for nursing home residents. Gerontologist 1992;32 (Special Issue II):254.

19. Spector WD, Fleishman JA. Combining activities of daily living with instrumental activities of daily living to measure functional disability. J Gerontol B Psychol Sci Soc Sci 1998;53B:S46-S57.

20. McFall S, Miller BH. Caregiver burden and nursing home admission of frail elderly persons. J Gerontol B Psychol Sci Soc Sci 1992;47B:S73-S79.

21. Morris J, Nonemaker S, Murphy K. A commitment to change: Revision of HCFA's RAI. J Am Geriatr Soc 1997;45:1011-1016.

22. Sullivan DH. The role of nutrition in increased morbidity and mortality. Clin Geriatr Med 1995;11:661-674.

23. Sullivan DH. Risk factors for early hospital readmission in a select population of geriatric rehabilitation patients: The significance of nutritional status. J Am Geriatr Soc 1992;40:792-798. 